\title{
Superior Neural Network for Distinguishing Between Atomic Species
}

Matthew Helmi Leth Larsen ${ }^{1}$, William Bang Lomholdt ${ }^{1}$, Anders Siig Dreisig ${ }^{1}$, Stig Helveg ${ }^{2}$, Ole Winther ${ }^{3}$, Thomas Hansen ${ }^{4}$ and Jakob Schiøtz ${ }^{5}$

${ }^{1}$ Technical University of Denmark, United States, ${ }^{2}$ Center for Visualizing Catalytic Processes (VISION), Department of Physics, Technical University of Denmark, DK-2800 Kgs. Lyngby, Denmark., Kongens Lyngby, Hovedstaden, Denmark, ${ }^{3}$ Department of Applied Mathematics and Computer Science, Technical University of Denmark, Kongens Lyngby, Hovedstaden, Denmark, ${ }^{4}$ DTU Nanolab, Technical University of Denmark, Kgs. Lyngby, Hovedstaden, Denmark, ${ }^{5}$ Department of Physics, Technical University of Denmark, Kongens Lyngby, Hovedstaden, Denmark

Identifying individual atoms in material samples is a challenging task in high-resolution transmission electron microscopy (HR-TEM) since the scattering of the incident electron beam can cause irreversible changes in the sample. This is an area where machine learning can step in to assist the process. Machine learning can analyze large amounts of data and provide fast detection of nanoparticles and atomic columns. We will here report advancements on the tailoring of deep learning neural networks for differentiating between individual atomic columns in molybdenum disulphide (MoS2) samples. The ability to simulate HR-TEM images for any given material allows for an extensive and detailed training process, utilizing amounts of data near impossible for a human operator to handle. This concept has previously been demonstrated for tracking gold atoms in HR-TEM image sequences [1] with an industry standard U-net architecture [2]. Training the network to distinguish between atomic species in multi-component material samples is possible by feeding the network a focal series of images. For example, providing three images of an MoS2 sample at three different defocus settings allows a neural network to differentiate between atomic columns consisting of either 1 molybdenum, 2 sulphur, or 1 sulphur atom in HR-TEM images. This work presents the superiority of the MSD-net [3], over the U-net architecture, in distinguishing between the described atomic columns in simulated HR-TEM images of MoS2. We use the F1-score as a measure for the performance of the networks, since it gauges the ability to avoid both false positives and false negatives. We have simulated HR-TEM images of bare MoS2 and MoS2 upon graphene substrates in the (001) crystal direction. A training set of 10,000 images and a validation set of 500 images has been simulated applying microscopy parameter values defined at random within a given range for each image. Succeeding this, we have trained an MSD-net architecture and a U-net architecture with the same training set and validated them using the same validation set. Figure 1 presents the inference (or predictions) of each network on the same simulated example of bare MoS2 from the validation dataset. Peaks are extracted from the inference and plotted against the simulated HR-TEM image. The U-net demonstrates overall more confusion and weakness in its predictions. Significantly weak areas are highlighted with yellow circles. The MSD-net, however, presents a near perfect prediction of all atomic columns. Figure 2 displays a histogram over the F1-score for each image in the validation set. This result shows that the MSD-net achieves higher F1-scores overall for the validation set of data proving its superiority over the U-net in predicting atomic columns correctly in HR-TEM images of MoS2. To improve the ability of the MSD-net in analyzing experimental images, we present a new method to include thermal vibrations in HR-TEM image simulations. This method consists of extracting a mean standard deviation of atomic positions dependent on their coordination number from a series of molecular dynamic simulations. These values are then used to perturb the atoms correctly by a simple translation of the positions. This is the frozen phonon approximation, but with vibrational amplitudes depending on the local structure obtained from molecular dynamic simulations. 
As a result, this includes thermal vibrational effects into the simulated images at a low computational cost. In conclusion, the MSD-net is shown to outperform the U-net in identifying atomic columns in multicomponent materials. This along with the computationally cheap implementation of thermal vibrations presents promising applications in the future of HR-TEM image segmentation.
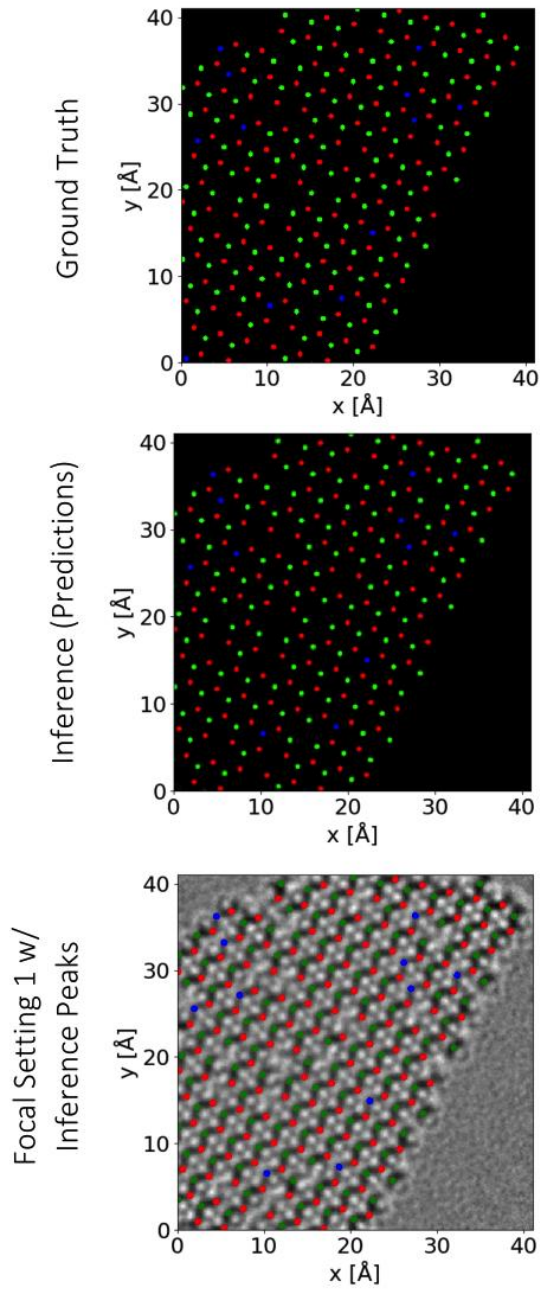

$1 \mathrm{Mo}$
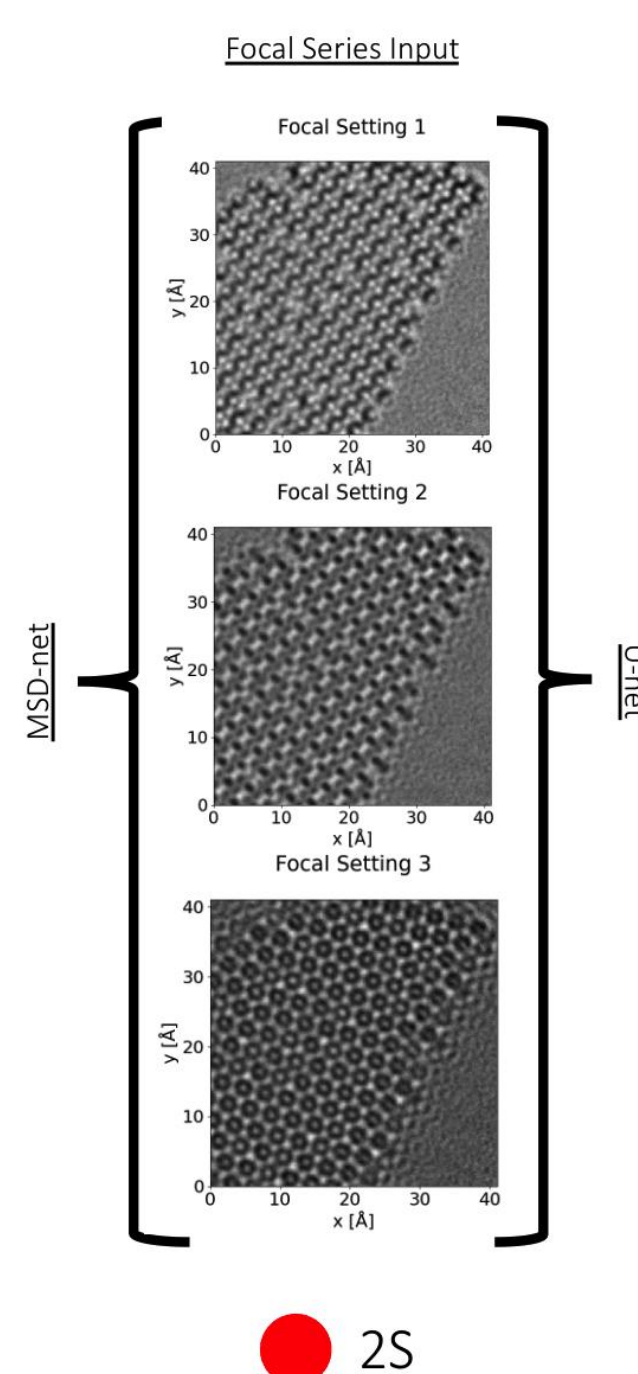
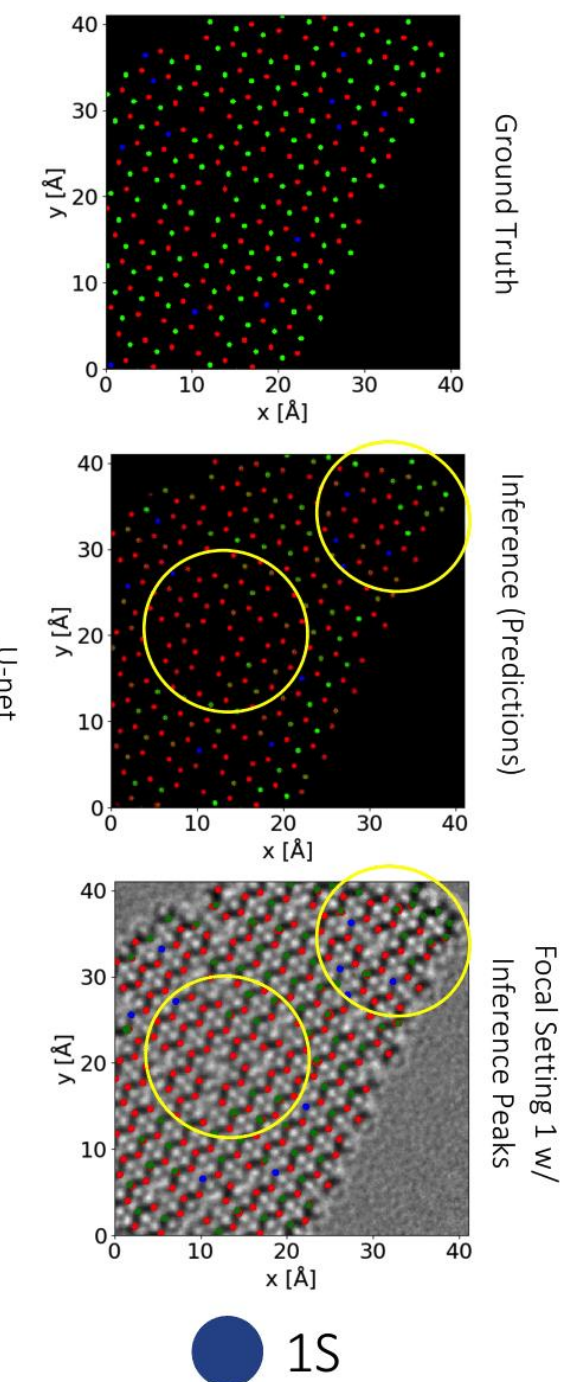

Figure 1. Qualitative presentation of predictions from LEFT: the MSD-net and RIGHT: the U-net on CENTRE: a simulated focal series of bare MoS2. The simulation is made with an electron beam acceleration voltage of $50 \mathrm{keV}$, a $3 \mathrm{rd}$ order spherical aberration coefficient of $14.29 \AA$, and a 1 st order astigmatism magnitude of $34.27 \AA$. Focal setting 1, 2, and 3 apply a defocus of $-30,20$, and $70 \AA$, respectively. The yellow circles highlight significantly weak areas in the predictions of the U-net. 

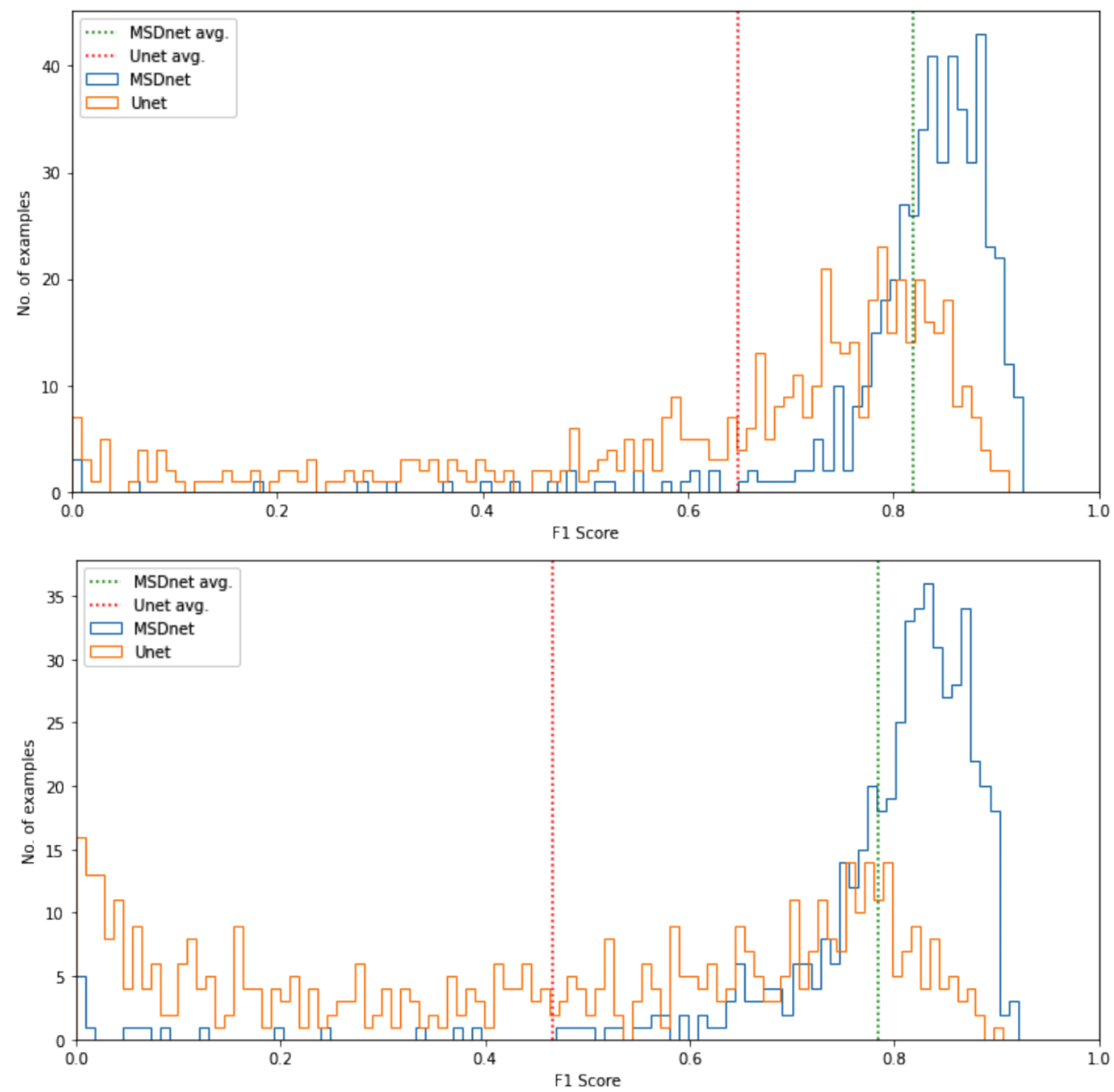

Figure 2. Histogram of F1-scores for predictions on a simulated validation set of $500 \mathrm{HR}$-TEM images of TOP: bare MoS2 and Bottom: MoS2 on graphene. Here we see the MSD-net's superior performance over the U-net in predicting the atoms by the higher average F1-score and overall higher F1-scores. It is also shown that the MSD-net does well at maintaining its performance when going from the bare MoS2 samples to the MoS2 samples supported on graphene.

\section{References}

1. Madsen, Jacob, et al. "A Deep Learning Approach to Identify Local Structures in Atomic-Resolution Transmission Electron Microscopy Images." Advanced Theory and Simulations, vol. 1, no. 8, Wiley-VCH Verlag, 2018, p. 1800037, doi:10.1002/adts.201800037.

2. Ronneberger, Olaf, et al. "U-Net: Convolutional Networks for Biomedical Image Segmentation." Lecture Notes in Computer Science (Including Subseries Lecture Notes in Artificial Intelligence and Lecture Notes in Bioinformatics), vol. 9351, Springer Verlag, 2015, pp. 234-41, doi:10.1007/978-3-319-24574-4_28.

3. Pelt, Daniël M., and Sethian, James A. "A Mixed-Scale Dense Convolutional Neural Network for Image Analysis." Proceedings of the National Academy of Sciences of the United States of America, vol. 115, no. 2, National Academy of Sciences, 2018, pp. 254-59. 